\title{
First Light on the Argonne PicoProbe and The X-ray Perimeter Array Detector (XPAD)
}

\author{
Nestor Zaluzec
}

\section{Argonne National Laboratory / Photon Science Directorate, Bolingbrook, Illinois, United States}

From the late 1960's to the 1990's X-ray Energy Dispersive Spectroscopy (XEDS) in the electron microscope was dominated by the Lithium Drifted Silicon Si(Li) detector [1-4] . For the last two decades these detectors have been slowly displaced by implementations of Silicon Drift Detectors SDDs [512]. During this time SDD performance with respect to drift, deadtime, and resolution has continuously improved and they have become routine ancillary x-ray detectors in most new implementations. The early work at Argonne, focused on interfacing SDD's to Analytical Electron Microscopes (AEMs), the first, a prototype windowless SDD system, was to the ANL HB603Z 300kV instrument. By today's XEDS standards, not only was the use of a SDD novel at that time, it also benefited by the fact our custom SDD had a large active area $\left(50 \mathrm{~mm}^{2}\right)$ and was relatively thick $(1 \mathrm{~mm})$. Both of which improved the solid angle and the high x-ray energy ( $>20 \mathrm{keV}$ ) detection efficiency. At that time because of its size and proximity to the specimen, the system attained a solid angle $\sim 0.45 \mathrm{sR}$. This was a improvement over the previously installed $\mathrm{Si}(\mathrm{Li})$ detector on the $\mathrm{HB} 603 \mathrm{Z}$ which reached $\sim 0.3 \mathrm{sR}$. Since that time, in order to generally improve the geometrical collection efficiency (and thus increase the available signal) various configurations of $\mathrm{x}$-ray detectors have become available which range from single large $\left(60->150 \mathrm{~mm}^{2}\right)$ to multiple small SDD sensors. In all cases, the objective has been to increase the effective solid angle and thus the measurable signal/nA [10-14], the largest of which to date has been the $\pi$ steradian detector [15]. Today, a wide range of implementations are commercially available, however, all are constrained by limits imposed by having to engineer around existing technology.

Since 2004, Argonne has been working on designs of linear arrays of SDD's in order to maximize the solid angle as well as mitigate artifacts. Evolving the $\pi$ steradian detector solution, the X-ray Perimeter Array Detector (XPAD) combined with a custom electron optical pole piece (ZTwin) has improved upon the $\pi$ steradian detector performance and is operating in the prototype instrument which is located in the Materials Design Laboratory at Argonne. PicoProbe locally achieved First Light on December 17th of 2020. In order to provide a specimen independent and reproducible evaluation of XEDS performance we employ a commercially available test specimen which is a $20 \mathrm{~nm}$ thick ultra-nanocrystalline Germanium film sputter deposited onto a $20 \mathrm{~nm}$ thick SiNx microporous self-supporting $0.5 \mathrm{~mm}^{2}$ window on $100 \mu \mathrm{m}$ thick Silicon disc [17]. This flat uniform test specimen has been described previously and allows robust testing and performance assessment of x-ray detectors in all analytical electron microscopes [18].

Figure 1a compares experimental results of the Ge Ka signal (integrated full peak intensity / $\mathrm{nA}-\mathrm{sec}$ ) from $\mathrm{Ge} / \mathrm{SiNx}$ test specimens for three different instruments all operating at $200 \mathrm{kV}$ :

. $\quad$ the Argonne Tecnai F20 with a Single $60 \mathrm{~mm}^{2}$ SDD

. $\quad$ the Argonne Talos F20x with Quad $30 \mathrm{~mm}^{2}$ SDD

- the Argonne Analytical PicoProbe with the XPAD

Using the $60 \mathrm{~mm}^{2}$ detector as a reference base $(1 \mathrm{x})$, we have found that the $X P A D$ improvement is greater than 20 times the single SDD system and exceeds the performance metrics of the original $\pi$ steradian detector, reaching Argonne's design target. Numerically, the performance of these three systems achieve the following metrics for a flat untilted Ge/SiNx specimen in optimized specimen holders and are characterized by the following integrated $\mathrm{Ge} \mathrm{K} \alpha$ intensity/nA-sec:

- Single $60 \mathrm{~mm} 2 \sim 600 \mathrm{cnts} / \mathrm{nA}-\mathrm{sec}$

- $\quad$ Quad $30 \mathrm{~mm} 2 \sim 2100 \mathrm{cnts} / \mathrm{nA}-\mathrm{sec}$ 
- Argonne XPAD $13500 \mathrm{cnt} / \mathrm{nA}$-sec

Due to the increased collection efficiency of the $X P A D$, there is also a corresponding improvement in the minimum detectable mass fraction. Illustrated in Figure $1 \mathrm{~b}$, are two experimental measurements both done of gold nanoparticles drop cast onto a $20 \mathrm{~nm}$ thick SiNx support membrane at $80 \mathrm{kV}$. The solid lower curve was measured using the Quad $30 \mathrm{~mm}^{2}$ detector system, while the upper dashed line was measured using the $X P A D$. An improvement of an order of magnitude (from $\sim 2 \times 10^{-3}$ to $\sim 2 \times 10^{-4}$ ) has been realized as was predicted [19]. In addition, the functional dependence of the $\mathrm{x}$-ray signal as well as the peak to background with incident beam energy from 30 to $300 \mathrm{keV}$, as discussed in that earlier work has also been verified.

Judicious design of collimation and shielding minimizes systems peaks, however they are not completely eliminated due in part to the large subtending solid angle of the XPAD. These peaks which result from peripheral scattering events are difficult to completely mitigate due to the optimization of the collection solid angle, however they are minimized with appropriate design effort.

Lastly, penumbra effects are paramount in limiting the performance of XEDS detectors in all AEMs, particularly due to the physical constraints imposed by specimen holders and their respective detector configurations which vary in all microscopes. Figure 2 compares the experimentally measured (and appropriately normalized) response of the three configurations when an optimized holder is used with the $\mathrm{Ge} / \mathrm{SiNx}$ test specimens. Again the XPAD demonstrates a superior functionality. This substantial improvement in performance with holder tilt, augments the improved solid angle and MMF results and also provides an improvement path for both quantification (by mitigating the need for tilting and thus absorption corrections) as well as enhancing the ability to measure tomographically resolved elemental distributions.

Additional work to delineate the performance metrics [20], has quantitatively measured the experimental solid angle to be $4.55+0.3 \mathrm{sR}$ at $300 \mathrm{kV}$ [21]. 

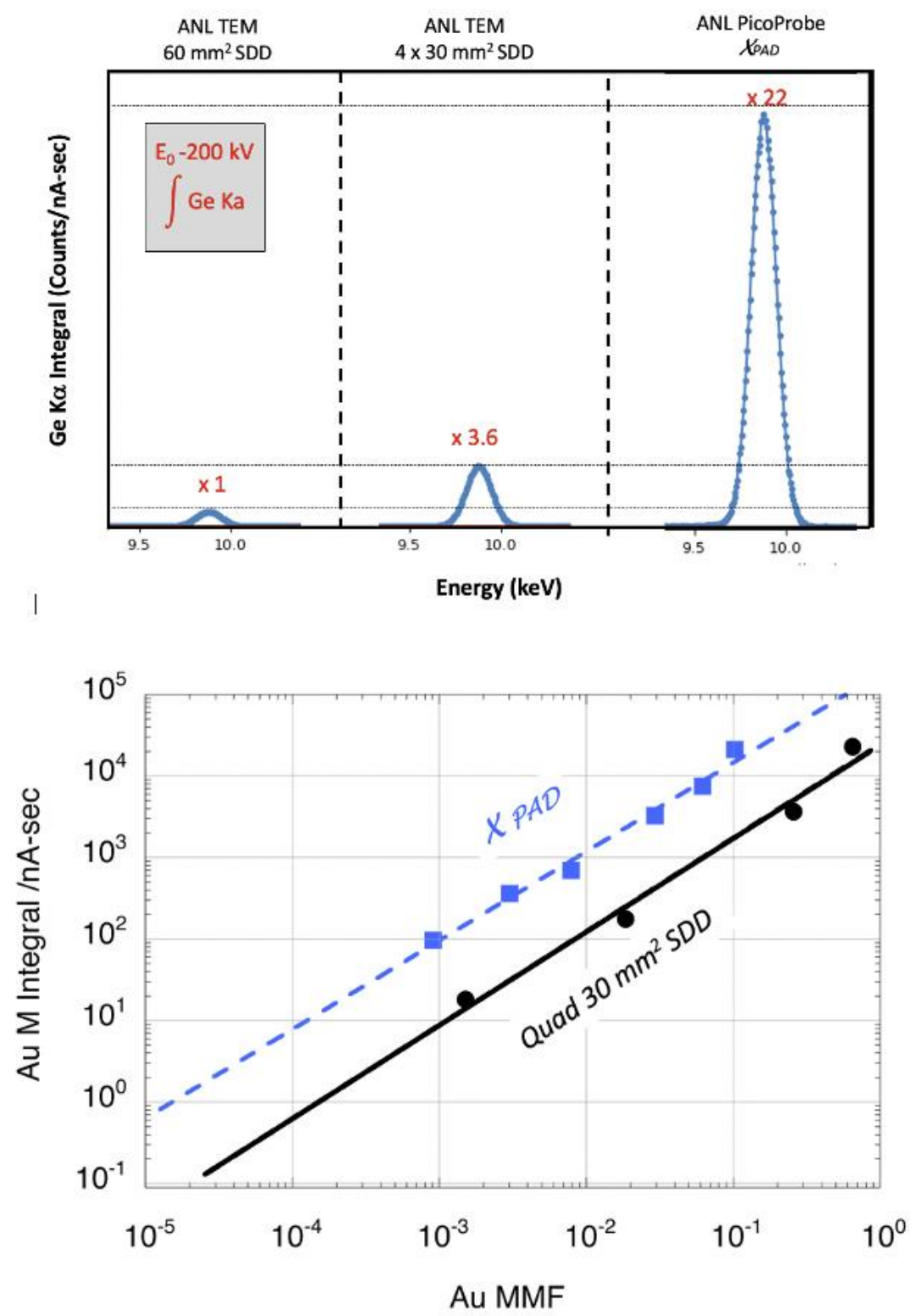

Figure 1. (a) Normalized performance of XEDS Collection for Ge Ka (Integrated Counts/nA-sec) from a 20nm thick ultra-nanocrystalline Ge film on SiNx for Single, Quad and XPAD systems. (b) Improvement of MMF of Au nanoparticles on SiNx at 80 kV. Solid line Quad 30 mm2 SDD, Dashed line XPAD. 


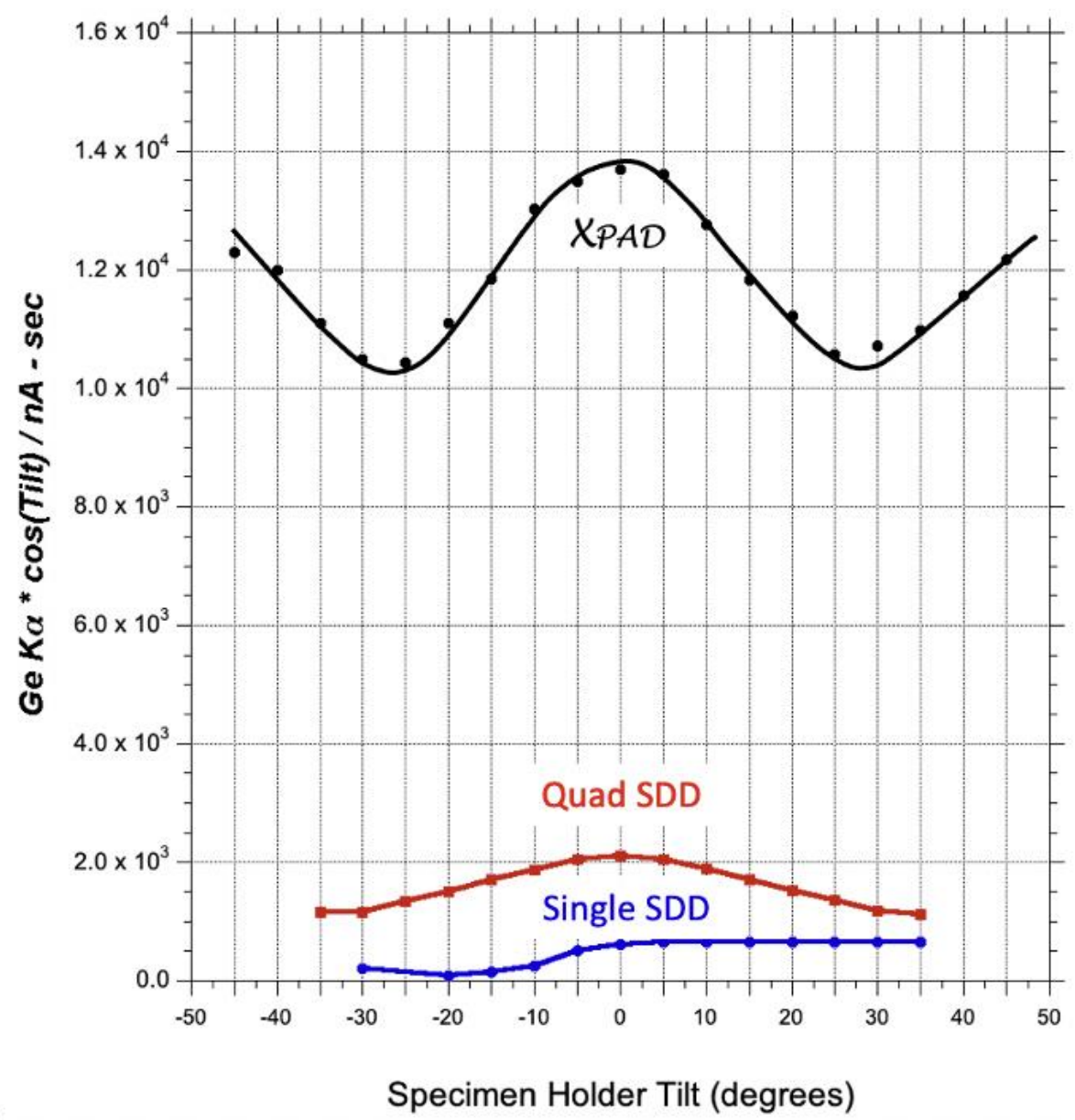

Figure 2. Experimental Penumbra effects as a function of specimen holder tilt for Argonne Instruments: Single $60 \mathrm{~mm} 2 \mathrm{SDD}$, Quad $30 \mathrm{~mm} 2 \mathrm{SDD}$ and XPAD

\section{References}

[1] Fitzgerald R., Keil K. \& Heinrich K.F.J. ;(1968). Science 159(3814), 528-530.

[2] Lorimer G.W., Razik N.A. \& Cliff G. ; (1973). J Microsc 99, 153-164.

[3] Zaluzec,N.J. ; (1991) Proc. of Microbeam Analysis Society, San Francisco Press, 137

[4] Lyman C.E., Goldstein J.I.,Williams, D.B., Ackland, D.W., von

H.S., Nicholls A.W., Statham, P.J.; (1994) J Microsc 176, 85

[5] Gatti E., Rehak. P,; (1984), Nucl. Instr. And Meth. In Phys. Res. 225608.

[6] Chen W. , et al, (1992) ; IEEE Trans. on Nucl. Sci. V39, 619.

[7] Iwanczyk J.S.,etal ; (1996) , Nucl. Instr. \& Meth. in Phys. Res. A380 (1996) 288

[8] Iwanczyk J.S., Patt B.E., Tull C.R., Barkan S., (2001), , Micro. Microanal. 7, S2 1052

[9] Barkan S., etal . ; (2004) ; Microscopy Today, 12, (6), 36,

[10] Watanabe, M., Wade, C.A. (2013). , Microsc Microanal 19, S2, 1264

[11] Tordoff, B., Beam, S., Schweitzer, M., Hill, E., Kugler, V. \& Png, K. (2012). Proc. EMC-2012, Manchester, September, PS2.2.

[12] von Harrach, H.S., Dona, P., Freitag, B., Soltau, H., Niculae, A. \& Rohde, M. (2009). Microsc Microanal 15(S2), 208-209.

[13] Zaluzec N.J.; (2004), Micro. Microanal., 10, S2, 122

[14] Zaluzec, N.J. (2009). Microscopy Today 17(4), 56. 
[15] Argonne National Laboratory. (2010). US Patent 8,314,386,

[16] See the WWW sites of leading manufacturers of electron microscopes and x-ray systems.

[17] http://www.temwindows.com/category_s/55.htm

[18] Zaluzec N.J., Wen J., Wang J., Miller D.J.; (2016), Microsc. Microanal. 22 ,S3 , 278-

[19] Zaluzec N.J. (2019) Ultramicroscopy V203 163

[20] Zaluzec N.J. ; Proc. of EMAG 2021, Manchester UK July 2021 in press

[21] Acknowledgements; This work was supported by the Photon Science Directorate and Laboratory Directed Research and Development (LDRD) funding from Argonne National Laboratory, provided by the Director, as well as the Office of Science, of the U.S. Department of Energy under Contract No. DEAC02-06CH11357. It was also supported in part by CRADA \#01300701 between Argonne National Laboratory and ThermoFisher Scientific Instruments. 